\title{
Autoimmune hemolytic anemia presenting concurrently with thrombotic thrombocytopenic purpura
}

\author{
Munaf AL-Kadhimi, Fernanda Wah, Tamarah AL-Dawoodi, \\ Julio Peguero, Luis T. Campos
}

\begin{abstract}
Thrombotic thrombocytopenic purpura (TTP) is an acute, life-threatening syndrome typically characterized by thrombocytopenia and microangiopathic hemolytic anemia (MAHA). Both genetic and idiopathic TTP are caused by deficient activity of the serine protease ADAMTS-13, allowing its normal substrate, von Willebrand factor, to polymerize and bind platelets under high sheer conditions. Autoimmune TTP is typically treated by plasmapheresis and corticosteroids. Here, we describe a 69-year-old patient presenting with thrombopenia comorbid with "warm" autoimmune hemolytic anemia (WAIHA). Treatment with steroids and plasma exchange initiated at the beginning and patient acquired a clinical as well as biological response. Thrombotic thrombocytopenic purpura (TTP) is a life-threatening condition typically (but not always) associated with autoimmune or genetic inactivation of the protease ADAMTS-13, for which consistently effective therapeutic interventions remain elusive.
\end{abstract}

Munaf AL-Kadhimi ${ }^{1}$, Fernanda Wah ${ }^{2}$, Tamarah AL-Dawoodi ${ }^{1}$, Julio Peguero ${ }^{1}$, Luis T Campos ${ }^{1}$

Affiliations: ${ }^{1} \mathrm{MD}$, Oncology Consultants, Research Department, International Cancer Center, Houston, TX; ${ }^{2} \mathrm{MD}$, Universidad Autonoma de Tampico, SN Matamoros, Zona Centro Ciudad Victoria, Tamaulipas, CP.

Corresponding Author: Dr. Julio Peguero MD, 2130 West Holcombe Blvd. 10th Floor, Houston, Texas 77030; E-mail: jpeguero@oncologyconsultants.com

Received: 25 July 2016

Accepted: 08 August 2016

Published: 16 August 2016
Keywords: Microangiopathic hemolytic anemia (MAHA), Thrombocytopenia, Thrombosis and hemolysis, Thrombotic thrombocytopenic purpura (TTP)

\section{How to cite this article}

AL-Kadhimi M, Wah F, AL-Dawoodi T, Peguero J, Campos LT. Autoimmune hemolytic anemia presenting concurrently with thrombotic thrombocytopenic purpura. Case Rep Int 2016;5:3639 .

Article ID: 100028CRINTMA2016

$$
* * * * * * * * *
$$

doi:10.5348/crint-2016-27-CR-9

\section{INTRODUCTION}

Thrombotic thrombocytopenic purpura (TTP) is a rare (U.S. prevalence of 1 in 68 , affecting $\sim 4$ million individuals) but life-threatening condition. TTP was first described by Dr. Eli Moschowitz in 1924 as a disease characterized by the unique pathological findings of hyaline thrombi in many organs, [1] later found to be largely composed of platelets [2]. A classic pentad of thrombocytopenia, microangiopathic hemolytic anemia (MAHA), fever, neurological symptoms, and renal abnormalities was described by Amorosi and Ultmann in 1966, present in 88-98\% of TTP patients at that time [3]. With the advent of plasmapheresis as a first-line treatment modality for TTP, and adoption of a lower threshold for initiation of treatment, the number of patients presenting with the classic pentad decreased to $40 \%$ in 1981 [4]. With the number of patients 
diagnosed with TTP up to 8-fold, and the increasing use of plasmapheresis early in the course of the disease, the number of patients who now present with the classic pentad is only 5\% [4]. Consequently, an essential dyad of thrombocytopenia and MAHA, in the appropriate clinical setting, is now the only requirement for establishing a diagnosis of TTP and initiating treatment [5].

It is now well established that idiopathic TTP is caused by autoantibodies against the serine protease ADAMTS-13, resulting in polymerization of its substrate, the clotting factor von Willebrand factor (vWF), while a more rare (<10\%) form, Upshaw-Schulman syndrome, results from recessively inherited, inactivating ADAMTS-13 mutations [6]. The result of downregulated ( $<10 \%$ of normal) ADAMTS-13 activity is the endothelial deposition of large, vWF multimers, causing sheer stress upon platelets within tissue microvessels, resulting in thrombosis and thrombocytopenia, in addition to sheering of erythrocytes and hemolysis, thus effecting anemia and schistocyte formation [5, 7]. The ensuing compromised blood flow, due to thrombosis and cellular injury, if untreated, results in end-stage organ damage [7]. Another condition, similar to TTP, warm autoimmune hemolytic anemia (WAIHA), is characterized by the production of autoantibodies directed against "self" RBCs, resulting in hemolysis, ranging in severity from mildly symptomatic to rapidly fatal [8].

As TTP is a medical emergency, suspected disease must be treated promptly [9]. While measurement of ADAMTS-13 activity is the best benchmark, its time to completion precludes its use as a sufficient diagnostic measure, upon patient presentation [10]. Consequently, following confirmation of the TTP dyad (via hematological assessment and blood smear), plasma exchange should be initiated as soon as possible [9]. Adjuvant or alternative therapies include corticosteroids, the B-cell antagonist (anti-CD20 antibody) rituximab, and other immunosuppressants, including cyclosporine and cyclophosphamide [11].

In this report, we describe a 69-year-old male presenting with an atypical case of TTP directly comorbid with WAIHA, our ensuing therapeutic interventions, and successful resolution of the pathology.

\section{CASE REPORT}

A 69-year-old Latin-American male presented at our emergency department with worsening confusion, slurred speech, and dizziness over the previous two weeks. On physical examination, the patient had stable vital signs, but generalized pallor, including skin, conjunctiva, palms, and nail beds. Neurological evaluation showed time disorientation and spontaneous speech with phonemic errors. His reading comprehension was limited to short words only, and he demonstrated minimal left upper extremity proximal paresis, deltoid $4+/ 5$.
Hematologic evaluation revealed a $4+$ pan reactive warm autoantibody [12] detected by elution, hemoglobin of $9.1 \mathrm{~g} / \mathrm{dl}$, and a platelet count of 16,000 . A peripheral smear showed spherocytes and mild schistocytosis. His autoimmune profile was negative for antinuclear antibodies (ANA), and normal serum complement levels. A CT scan of the brain revealed a left posterior temporal hemorrhagic stroke.

Due to his manifestation of symptoms, the patient was admitted to the neurological intensive care unit, and within the first 12 hours, he was treated with steroids alone, but exhibited a complete lack of evidence of response, resulting in the suspicion of TTP. Subsequently, a four-liter plasma volume exchange approach was initiated within the first 12 hours of admission, and every 24 hours thereafter. Following a total of six sessions of plasma exchange, his platelet count showed improvement, increasing to 192,000. One week later his pretreatment ADAMTS-13 level was less than $3 \%$, and he was then discharged and referred for outpatient neurological rehabilitation. His progress continued, as his serial ADAMTS-13 levels recovered to optimal range, and platelet levels remained within their normal range. The warm antibody faded out with the continuum of treatment, and his peripheral smear cleared. Neurologically, at this time, he is intact.

\section{DISCUSSION}

Thrombotic thrombocytopenic purpura (TTP) is an acute, life-threatening syndrome typically characterized by thrombocytopenia and microangiopathic hemolytic anemia (MAHA) [5]. There is often multi-organ involvement, with neurological and renal abnormalities being the most common and potentially, the most serious[10]. A classic pentad of thrombocytopenia, MAHA, fever, neurological abnormalities, and renal dysfunction has been described, but is rarely seen in its entirety in the present day, with a "dyad" of two major diagnostic criteria, thrombocytopenia and microangiopathic hemolytic anemia (MAHA), absent alternate explanation, now considered sufficient to suspect TTP and initiate treatment [5, 9]. In 1990, an association between (TTP) and autoimmune hematological (AIHA) conditions was reported in two patients. In one case, a 35-year-old male patients, suffered from AIHA for five years before the appearance of TTP. The other case was a 14-year-old female that developed the disease four years after the onset of AIHA [13]. Differential diagnoses include hemolytic uremic syndrome (usually juvenile), antiphospholipid syndrome, and systemic lupus erythematosus [14].

In this report, we present a unique case in which TTP was considered a differential diagnosis in a phenomena presenting concurrently with AIHA. Consequently, we decided to raise awareness of this distinct clinical presentation, and to emphasize that TTP can introduce 
itself with virtually any autoimmune disorder [15]. Contrary to some literature reports, these clinical presentations are not mutually exclusive, and TTP should be suspected even in the setting of a predominant population of spherocytes with mild schistocytes, [16] as evidenced by the profound deficiency of ADAMTS- 13 that affected our patient.

\section{CONCLUSION}

Thrombotic thrombocytopenic purpura (TTP) is a life-threatening condition typically (but not always) associated with autoimmune or genetic inactivation of the protease ADAMTS-13, for which consistently effective therapeutic interventions remain elusive. While yet considered a rare condition, TTP is likely more common than previously believed, and due to its urgency, should be promptly be suspected in cases presenting with thrombosis and hemolysis.

\section{$* * * * * * * * *$}

\section{Acknowledgements}

We wish to thank Julio Peguero M.D, Luis T Campos M.D for providing editorial support.

\section{Author Contributions}

Munaf AL-Kadhimi - Substantial contributions to conception and design, Acquisition of data, Analysis and interpretation of data, Drafting the article, Revising it critically for important intellectual content, Final approval of the version to be published

Fernanda Wah - Substantial contribution to conception and design, Analysis and interpretation of data, Drafting the article, Final approval of the version to be published Tamarah AL-Dawoodi - Substantial contribution to conception and design, Analysis and interpretation of data, Drafting the article, Revising it critically for important intellectual content, Final approval of the version to be published

Julio Peguero - Substantial contribution to conception and design, Analysis and interpretation of data, Final approval of the version to be published

Luis T Campos - Substantial contribution to conception and design, Analysis and interpretation of data, Final approval of the version to be published

\section{Guarantor}

The corresponding author is the guarantor of submission.

\section{Conflict of Interest}

Dr. Peguero serves in the speaker bureau for Foundation Medicine, Bristol Myers Squibb, and Bayer. $\mathrm{He}$ directs phase 1 and 2 clinical trials, funded by various pharmaceutical companies, within the Oncology Consultants Research Department. None of the remaining authors have any conflict of interest.

\section{Copyright}

(C) 2016 Munaf AL-Kadhimi et al. This article is distributed under the terms of Creative Commons Attribution License which permits unrestricted use, distribution and reproduction in any medium provided the original author(s) and original publisher are properly credited. Please see the copyright policy on the journal website for more information.

\section{REFERENCES}

1. Moschcowitz E. Hyaline thrombosis of the terminal arterioles and capillaries: A hitherto undescribed disease. Proc N Y Pathol Soc 1924;24:21-4.

2. Asada Y, Sumiyoshi A, Hayashi T, Suzumiya J, Kaketani K. Immunohistochemistry of vascular lesion in thrombotic thrombocytopenic purpura, with special reference to factor VIII related antigen. Thromb Res 1985 Jun 1;38(5):469-79.

3. Amorosi E, Ultmann JE. Thrombotic thrombocytopenic: Report of 16 cases and review of the literature. Medicine 1966;45(2):139-60.

4. Ridolfi RL, Bell WR. Thrombotic thrombocytopenic purpura. Report of 25 cases and review of the literature. Medicine (Baltimore) 1981 Nov;60(6):41328.

5. Said A, Haddad RY, Stein R, Lerma EV. Thrombotic thrombocytopenic purpura. Dis Mon 2014 Oct;60(10):500-4.

6. Galbusera M, Noris M, Remuzzi G. Inherited thrombotic thrombocytopenic purpura. Haematologica 2009 Feb;94(2):166-70.

7. Moake JL. von Willebrand factor, ADAMTS-13, and thrombotic thrombocytopenic purpura. Semin Hematol 2004 Jan;41(1):4-14.

8. Naik R. Warm autoimmune hemolytic anemia. Hematol Oncol Clin North Am 2015 Jun;29(3):44553.

9. Kessler CS, Khan BA, Lai-Miller K. Thrombotic thrombocytopenic purpura: A hematological emergency. J Emerg Med 2012 Sep;43(3):538-44.

10. Zheng XL. ADAMTS13 and von Willebrand factor in thrombotic thrombocytopenic purpura. Annu Rev Med 2015;66:211-25.

11. Sayani FA, Abrams CS. How I treat refractory thrombotic thrombocytopenic purpura. Blood 2015 Jun 18;125(25):3860-7.

12. Morgensztern D, Kharfan-Dabaja MA, Tsai HM, Lian EC. Warm-antibody autoimmune hemolytic anemia developing after thrombotic thrombocytopenic purpura. Acta Haematol 2002;108(3):154-6.

13. Krupsky M, Sarel R, Hurwitz N, Resnitzky P. Late appearance of thrombotic thrombocytopenic purpura after autoimmune hemolytic anemia and in the course of chronic autoimmune thrombocytopenic purpura: two case reports. Acta Haematol 1991;85(3):139-42.

14. Veyradier A, Meyer D. Thrombotic thrombocytopenic purpura and its diagnosis. J Thromb Haemost 2005 Nov;3(11):2420-7.

15. John ML, Scharrer I. Autoimmune disorders in patients with idiopathic thrombotic thrombocytopenic purpura. Hamostaseologie 2012;32 Suppl 1:S86-9. 
16. Reardon JE, Marques MB. Evaluation of Thrombocytopenia. Lab Med 2006;37(4):248-50.

Access full text article on other devices

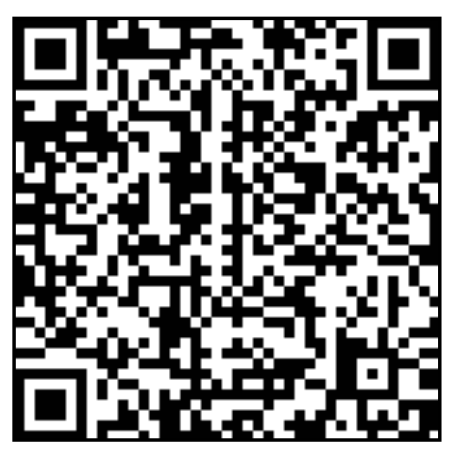

Access PDF of article on other devices

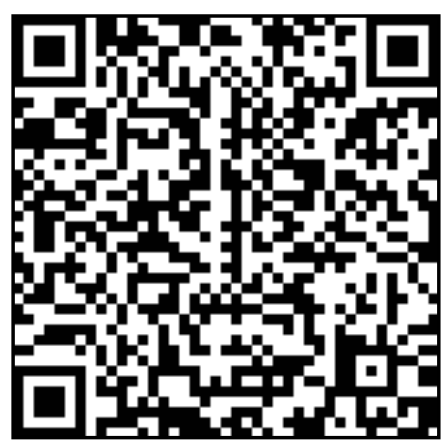

